\title{
Macroscopic Device Simulation of InGaAs/InP Based Avalanche Photodiodes
}

\author{
JOSEPH W. PARKS, KEVIN F. BRENNAN and LARRY E. TAROF \\ Microelectronics Research Center, Georgia Institute of Technology Atlanta, GA USA 30332-0269 \\ Bell Northern Research,P.O. Box 3511 Station C Ottawa, Canada K1Y4H7
}

\begin{abstract}
In this paper, we analyze, based on a two-dimensional drift-diffusion simulation, how variations in the structural components of an InGaAs/InP separate absorption, grading, charge, and multiplication photodiode (SAGCM) alter its performance. The model is employed in conjunction with experimental measurements to enhance the understanding of the device performance. Calibration of the model to the material system and growth technique is performed via the analysis of a simpler, alternate structure. Excellent agreement between the calculated results and experimental measurements of the breakdown voltage, dark current, mesa punchthrough voltage, photoresponse, and gain are obtained.
\end{abstract}

\section{INTRODUCTION}

The SAGCM APD has been shown to be an effective detector design for light-wave communication purposes[1,2]. The design combines the attractive features of high gain, large bandwidth, and relative relaxation of fabrication tolerances. A cross section of the basic structure under consideration is shown in the inset of Figure 1. It is evident that the underlying geometry of this device is a akin to a "lo-hi-lo" Read diode. The charge sheet, used to control the electric field profile, is divided into two parts in order to prevent premature breakdown at the edge of the $p+$ diffusion. The lattice matched InGaAs absorption layer is separated from the InP via a linearly graded region in order to prevent hole trapping. The calculated nominal photo-illuminated and dark currents for this device are given in Figure 1. From the figure, it is clear that there exists three primary modes of operation for this device. At low bias indicated by Region I, only a portion of the InP region has been depleted, and subsequently, the photoresponse is negligible. When the device is biased into Region II, the annular portion of the charge sheet is depleted as well as a portion of the InGaAs directly behind the annulus. The device now performs similarly to a $\mathrm{p}$-i-n diode and the dark current is dominated by thermal generation in the InGaAs. At high reverse bias, Region III, the mesa charge sheet has also been fully depleted. Thermally generated or photoexcited carriers may now drift from the InGaAs directly into the InP by passing through the high-field multiplication region. Provided that the electric field is sufficiently large in the multiplication region, the carriers will undergo avalanche multiplication and the device behaves as an APD as desired. 


\section{MODEL DESCRIPTION AND CALIBRATION}

In modeling the SAGCM APD, the drift-diffusion subset of our two-dimensional simulator, STEBS-2D [3], was used. Poisson's equation, the current continuity of both carriers, and the flux equations for both carriers given by:

$$
\begin{aligned}
& \begin{array}{r}
\nabla\left(\varepsilon_{r} \nabla \Psi\right)=\frac{q}{\varepsilon_{o}}\left(p-n+N_{d}^{+}-N_{a}^{-}\right) ; \\
\left(\frac{\partial n}{\partial t}\right)+\nabla \cdot(n \bar{v})=G-R
\end{array} \\
& n \bar{v}=\mu k_{b} T_{0} \nabla n+n \mu \nabla \varepsilon_{c}-n \mu \nabla x+n \mu k_{b} T_{0} \frac{\nabla N_{c}}{N_{c}}
\end{aligned}
$$

are solved self-consistently to determine the potential and carrier concentration within the simulation domain. Auger, radiative band-to-band, and Shockley-Read-Hall mechanisms are included within the recombination-generation rates. Additionally, an exponential photogeneration rate and a Chynoweth impact ionization rate are also included[4]. The carrier mobilities follow standard field dependent expressions which account for negative differential mobility. Heterostructures and material non-uniformity are also included in the model. A thermionic emission boundary condition is used at heterointerfaces to insure that proper current limitations are enforced[5]. Table I enumerates some of the parameters used in

\begin{tabular}{|c|c|c|c|}
\hline Parameter & Units & $\operatorname{In} G a A s$ & $\operatorname{In} P$ \\
\hline Bandgap & $(\mathrm{eV})$ & 0.77 & 1.34 \\
\hline Electron Affinity & $(\mathrm{eV})$ & 4.7 & 4.51 \\
\hline Relative Permittivity & & 13.85 & 12.61 \\
\hline Electron Mass (relative) & & 0.0463 & 0.08 \\
\hline Heavy Hole Mass (relative) & & 0.61 & 0.85 \\
\hline Light Hole Mass (relative) & & 0.0588 & 0.089 \\
\hline Electron Mobility $\left(\mu_{10}\right)$ & $\left(\mathrm{cm}^{2} / \mathrm{Vs}\right)$ & 12000 & 4730 \\
\hline Electron Saturation Velocity $\left(\mathrm{v}_{\text {sat }}\right)$ & $(\mathrm{cm} / \mathrm{s})$ & $7 \mathrm{e} 6$ & $2.6 \mathrm{e} 7$ \\
\hline Electron Critical Field $\left(\mathrm{E}_{\text {crit }}\right)$ & $(\mathrm{V} / \mathrm{cm})$ & 3000 & 3500 \\
\hline Hole Mobility $\left(\mu_{10}\right)$ & $\left(\mathrm{cm}^{2} / \mathrm{Vs}\right)$ & 450 & 151 \\
\hline Hole Saturation Velocity $\left(\mathrm{v}_{\text {sat }}\right)$ & $(\mathrm{cm} / \mathrm{s})$ & $4.9 \mathrm{e} 6$ & $2.6 \mathrm{e} 7$ \\
\hline Electron Auger Coeff. $\left(C_{n}\right)$ & $\left(\mathrm{cm}^{6} / \mathrm{s}\right)$ & $3.2 \mathrm{e}-28$ & $3.7 e-31$ \\
\hline Band-to-Band Coeff (B) & $\left(\mathrm{cm}^{3} / \mathrm{s}\right)$ & $5.4 \mathrm{e}-11$ & $3.23 e-10$ \\
\hline Electron SRH lifetime $\left(\tau_{\mathrm{n} 0}\right)$ & $(\mathrm{sec})$ & $2.2 \mathrm{e}-4$ & $1.0 \mathrm{e}-9$ \\
\hline Hole Auger Coeff. $\left(C_{n}\right)$ & $\left(\mathrm{cm}^{6} / \mathrm{s}\right)$ & $3.2 \mathrm{e}-28$ & $8.7 e-30$ \\
\hline Hole SRH lifetime $\left(\tau_{\mathrm{n} 0}\right)$ & $(\mathrm{sec})$ & $2.2 \mathrm{e}-4$ & $1.0 \mathrm{e}-9$ \\
\hline Electron Impact Ionization $\alpha_{0}$ & $\left(\mathrm{~cm}^{-1}\right)$ & $4.677 \mathrm{e} 7$ & $1.12 \mathrm{e} 7$ \\
\hline Electron II Critical Field $\left(\mathrm{E}_{\text {crit }}\right)$ & $(\mathrm{V} / \mathrm{cm})$ & $1.935 \mathrm{e} 6$ & $3.11 \mathrm{e} 6$ \\
\hline Hole Impact lonization $\alpha_{0}$ & $\left(\mathrm{~cm}^{-1}\right)$ & $8.382 \mathrm{e} 7$ & $4.79 \mathrm{e} 6$ \\
\hline Hole II Critical Field ( $\left.\mathrm{E}_{\text {crit }}\right)$ & $(\mathrm{V} / \mathrm{cm})$ & $2.236 \mathrm{e} 6$ & $2.55 \mathrm{e} 6$ \\
\hline Light absorption Coeff, $\alpha(\lambda=1.55 \mu \mathrm{m})$ & $\left(\mathrm{cm}^{-1}\right)$ & 8000 & 0 \\
\hline
\end{tabular}
these simulations.

TABLE I List of material parameters for InP and InGaAs used for all SAGCM simulations. 


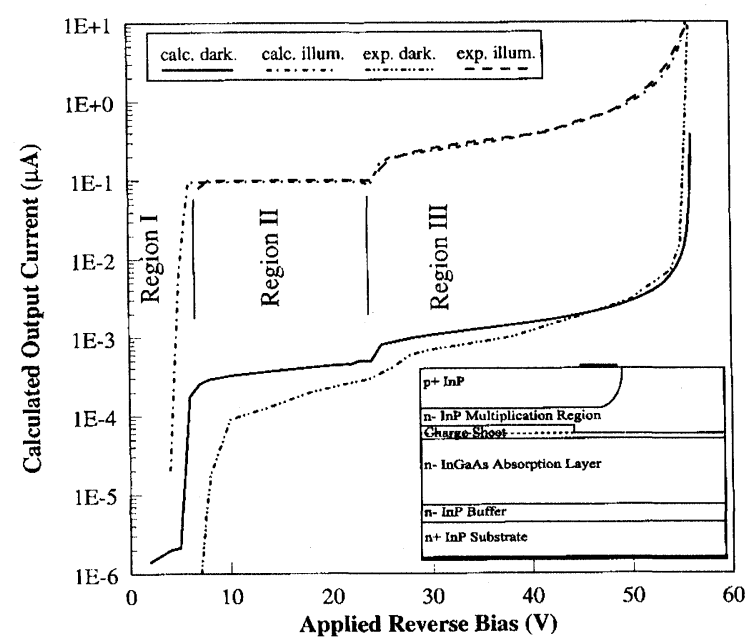

FIGURE 1 Calculated and experimental steady-state current-voltage response for the SAGCM device under dark and illuminated conditions. Inset shows basic device geometry

As with any numerical model, the calibration of the model to the material system and growth technique at hand is critical. Even though the majority of material parameters were obtained through literature searches, we felt it necessary to calibrate our model with a similar device manufactured using the same fabrication processes. In particular the low-level injection lifetime, $\tau_{S R H}$, which could vary with growth technique, was obtained. The device under consideration is also

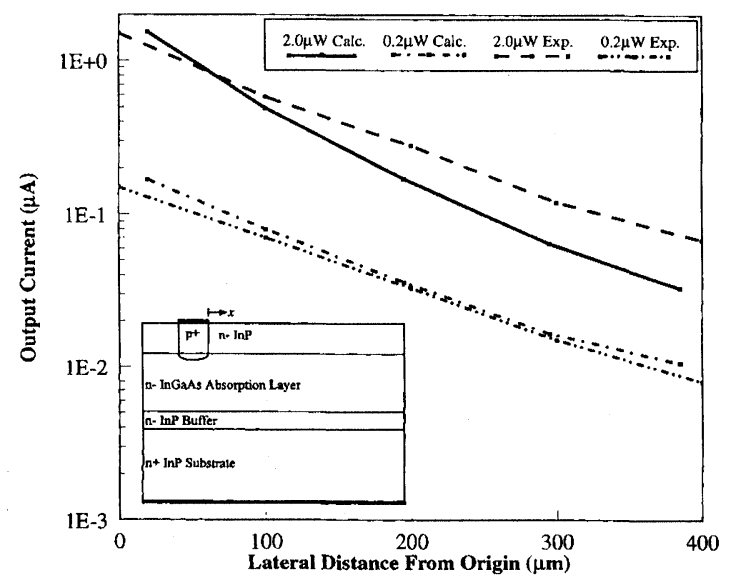

FIGURE 2 Current response of the p-i-n diode as a function of the lateral distance between the light source and the edge of the $p+$ diffusion profile. Using simulations of this device, the model was calibrated with experimental results for the low-level injection lifetime for electrons in the InGaAs material system a double-heterostructure InP/InGaAs/InP device, however, it operates as a p-i-n diode [6]. The device is photo-excited from the top surface by a laterally moving source. Since the bias conditions are set such that high field effects are not present, a direct comparison of the diffusion length is possible. The device geometry along with a comparison of the experimental and numerically calculated currents are given in Figure 2. As seen in the figure, good quantitative agreement is found under low level injection. With the correct lifetimes now accurately set, the examination of the more complex SAGCM structure was plausible.

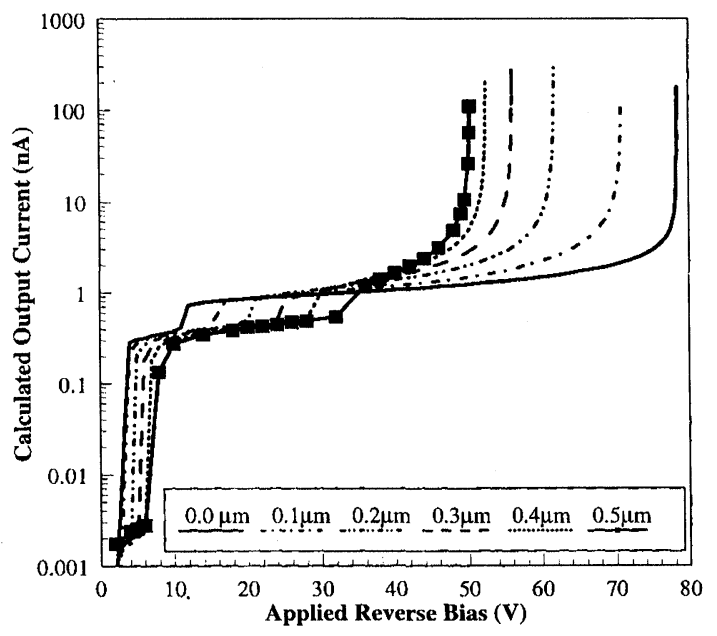

FIGURE 3 Family of curves showing the calculated output current versus voltage for various multiplication region thicknesses

\section{SIMULATION OF SAGCM DEVICE}

Using the previously described model, it is possible to successfully simulate the SAGCM APD. Good quantitative agreement with experimental measurements of the dark and photo-excited current is found[7] as seen in Figure 1. With the understanding that the model can accurately predict the baseline response of the device, additional characterization can be performed by systematically varying key geometrical components of the device. Figure 3 shows a sampling of this analysis indicating how perturbations in the multiplication region thickness, $x_{d}$, affect the dc response of the device. This indicates that relatively 
minor changes in the device geometry are seen to dramatically alter the range over which the device operates.

\section{CONCLUSIONS}

In conclusion, we have performed a theoretical study of the SAGCM avalanche photodiode in order to study how changes in the device geometry affect its overall performance. The drift-diffusion simulator employed in this study is calibrated to a similar $\mathrm{p}-\mathrm{i}-\mathrm{n}$ device using the same material system and growth technique through the low level injection lifetime parameter. Good agreement is obtained with the experimental current-voltage characteristics.

\section{Acknowledgements}

This work at Georgia Tech was sponsored in part by Bell Northern Research through contract E21-5D7.

\section{References}

11] L.E. Tarof, J. Yu, R. Bruce, D.G. Knight, T. Baird, and B. Oosterbrink, "High Frequency Performance of Separate Absorption, Grading, Charge, and Multiplication InP/InGaAs Avalanche Photodiodes," IEEE Photon. Technol. Letters, vol. 5, no. 6, pp. 672-674, 1993.

[2] L.E. Tarof, J. Yu, D.G. Knight, T. Baird, K. Visvanatha, D. McGhan, B. Emmerstorfer, C.L.F. Ma, C.M. Martin, C.J. Miner, "Design and Whole Wafer Performance of Separate Absorption, Grading, Charge, and Multiplication InP/ InGaAs Avalanche Photodiodes," SPIE Proceedings-Technologies for Optical Fiber Communications, vol. 2149, pp. 290-301,1994.

[3] A.W. Smith, "Light Confinement and Hydrodynamic Modeling of Semiconductor Structures Using Volumetric Techniques," Ph.D. Dissertation, Georgia Institute of Technology, 1992.

[4] S. Selberherr, "Analysis and Simulation of Semiconductor Devices,” Springer-Verlag, Wien, New York, 1984.

[5] K. Horio and H. Yanai, "Numerical Modeling of Heterojunction Including the Thermionic Mechanism at the Heterojunction Interface," IEEE Trans. Elec. Dev., vol. 36, no. 3, pp. 321-330, 1993.

[6] M. Gallant and A. Zemel, "Long Minority Hole Diffusion Length and Evidence for Bulk Radiative Recombination Limited Lifetime in InP/InGaAs/InP Double Heterostructure," Appl. Phys. Lett., vol. 52, no. 20, pp. 1686-1688, 1988.

[7] C. Ma, M. Deen, L. Tarof, J. Yu, "Temperature Dependence of Breakdown Voltages in Separate Absorption, Grading, Charge, and Multiplication InP/InGaAs Avalanche Photodiodes," IEEE Trans. Elec. Dev., vol.42, no. 5, pp. 810-818, 1995

\section{Biographies}

Joseph W. Parks Jr. was born in Oak Ridge, TN on May 21, 1970. He received his B.S. degree in electrical engineering from the University of Tennessee, Knoxville in 1992 and his M.S.E.E. from the Georgia Institute of Technology, Atlanta, GA, in 1993. He is presently working on his $\mathrm{Ph} . \mathrm{D}$. degree also in electrical engineering at the Georgia Institute of Technology. His research work involves the numerical modeling of semiconductor devices with emphasis in the drift-diffusion and hydrodynamic simulation of photodetectors and avalanche photodiodes.

Kevin F. Brennan received the B.S. degree in physics from the Massachusetts Institute of Technology, Cambridge, MA in 1978 and the M.S. degree in physics and Ph.D. degree in Electrical Engineering from the University of Illinois in Urbana-Champaign in 1984. He is currently Institute Fellow and Professor in the School of Electrical and Computer Engineering at Georgia Tech in Atlanta, Georgia. He is a recipient of a Presidential Young Investigator Award through the National Science Foundation. His current research interests include the physics and modeling of semiconductor devices. Of particular interest are the physics and modeling of avalance photodiodes, confined state ionization devices, high field effects in semiconductors, photoconductors, and high speed transistors.

Larry E. Tarof was born in 1960 . He received the B.Sc. degree from the University of Toronto in physics in 1983, and both the M.Eng. and the Ph.D. degrees from the Carleton University, Ottawa, Ontario, in electronics in 1989 and 1993, respectively. He has been at Bell-Northern Research, Ottawa, since 1984 as a member of scientific staff working on optoelectronic devices. He has developed new processing and analysis techniques for III-V materials, includin optical methods for nondestructive epitaxial layer thickness and composition measurement. His present research interests include device physics of optoelectronic devices, and avalanche photodiodes in particular. Dr. Tarof holds one patent and has published more than twenty papers or conference proceedings. 

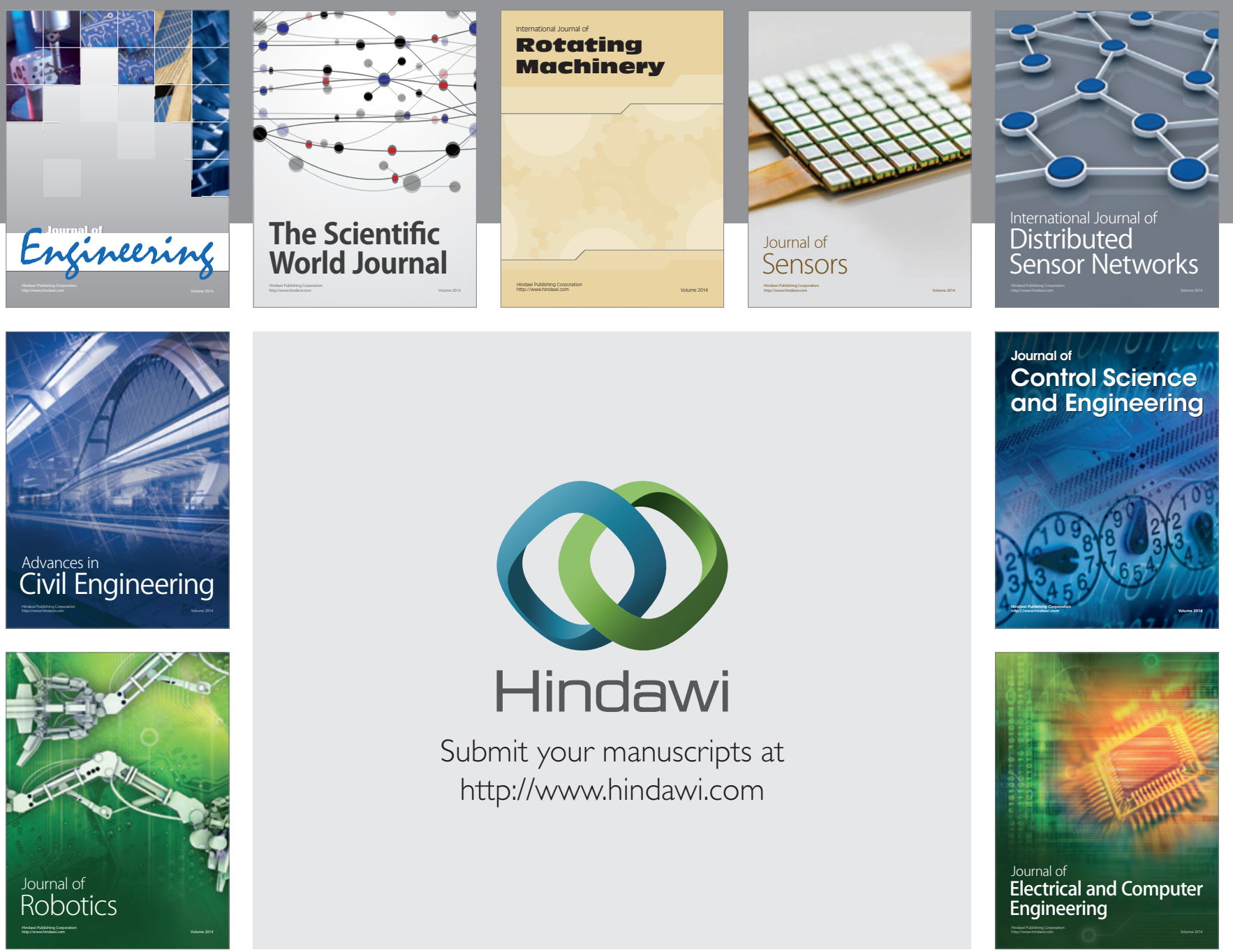

Submit your manuscripts at

http://www.hindawi.com
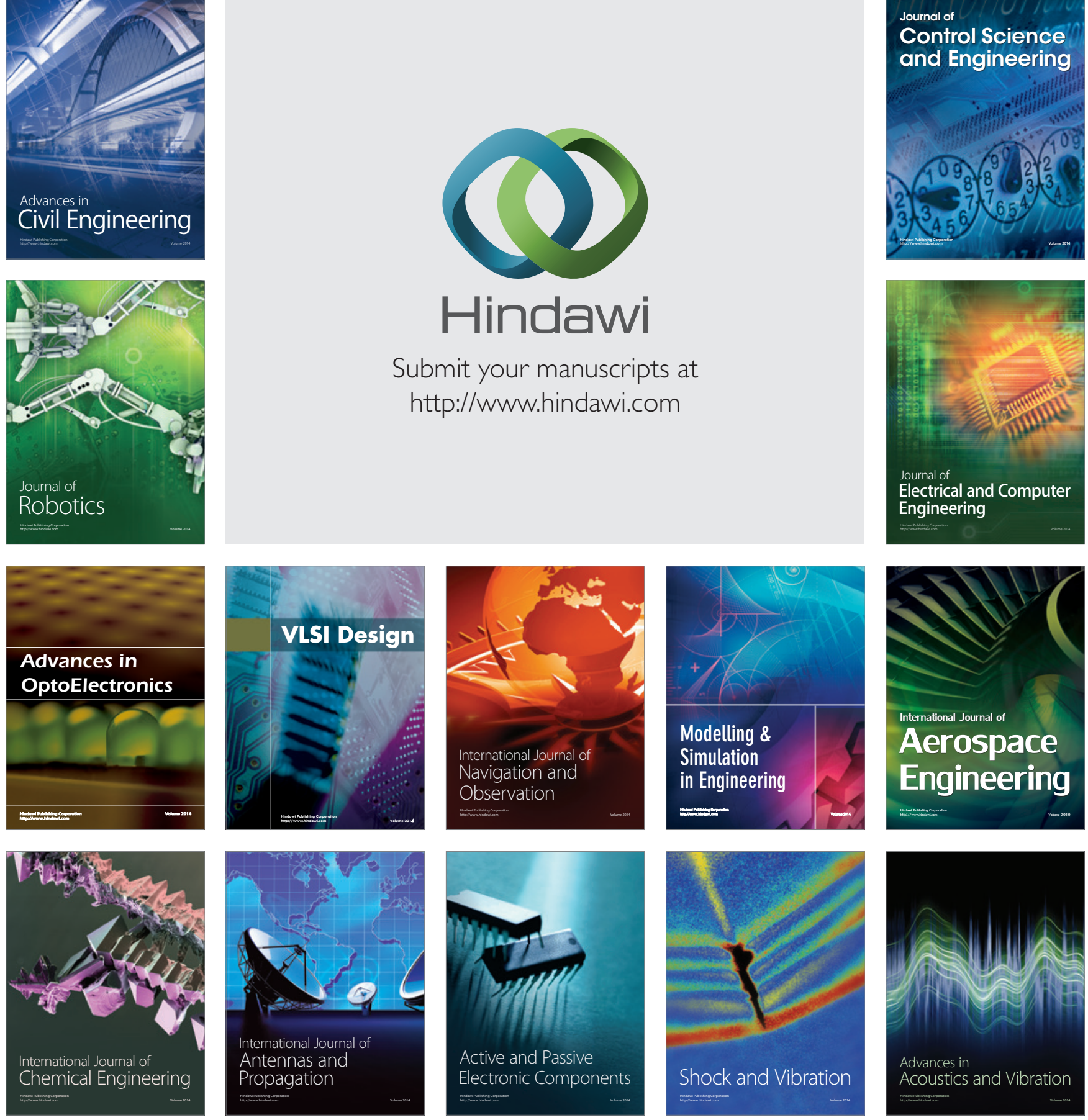